\title{
Coordinated Switching Control of the Excitation and Steam Valve System Based on State and Input Constraints
}

\author{
Nan Jiang, ${ }^{1}$ Ting Liu, ${ }^{2}$ Haiyan He, ${ }^{1}$ and $\mathrm{Xin}_{\mathrm{Li}}{ }^{1}$ \\ ${ }^{1}$ Faculty of Information Science and Engineering, Northeastern University, Shenyang, Liaoning 110819, China \\ ${ }^{2}$ Department of Electrical Engineering and Automation, College of Light Industry, Liaoning University, Shenyang 110036, China \\ Correspondence should be addressed to Ting Liu; liuting_tinka@sina.cn
}

Received 13 February 2015; Accepted 25 April 2015

Academic Editor: Bo Shen

Copyright (c) 2015 Nan Jiang et al. This is an open access article distributed under the Creative Commons Attribution License, which permits unrestricted use, distribution, and reproduction in any medium, provided the original work is properly cited.

\begin{abstract}
A single unit infinite system of the excitation and steam valve control was proposed based on Barrier Lyapunov theory of restrictive log type. The input amplitude constraint of the steam valve control was considered, and the coordinated nonlinear backstepping controller was designed by switching mechanism. At the same time, the generator rotor effect was considered to be an external unknown large disturbance on the output system, and the conservativeness of the simple estimates for the upper and lower bounds and scaling disturbance was reduced by Minimax. The Minimax method also ensured that the output of the controller and the power angle were within the prescribed range and inhibited the system output effect of disturbance as much as possible. Finally, simulation results of the generator disturbance of mechanical power in the single unit infinite system show that the control scheme effectively improves the transient stability of the dynamic processes of power systems.
\end{abstract}

\section{Introduction}

Turbine generator excitation control and steam valve control are two key methods for improving the stability of power systems. The rising speed of the excitation current is limited to the constant of the field winding, and excitation control is limited to the maximum value of the excitation current (the maximum value of the excitation current of a generator is generally 1.8 up to 2.0 times its rated current). Requiring excessive maximum value excitation current will increase the manufacturing costs of generators [1], and relying just on excitation control to enhance system stability results in limited improvement.

A prime mover using nonlinear control for opening the steam valve is often effective for further improving the transient stability of power systems. In view of the large power reheat turbine generator sets used in power systems, mechanical hydraulic governors are increasingly being replaced by power-frequency electrohydraulic governors. The capacity and load adaptability of the primary frequency are improved by the opening steam valve control in the reheat turbine generator set. Thus, an increasing number of engineering researchers are focusing on the stability of power systems.
Many advanced control methods have been recently applied to the excitation and steam valve controller design of generators. Reference [2] described a nonlinear model of power systems that conducts precise linearization of state variable feedback. In this model, the nonlinear controller of the opening steam valve was designed by differential geometry method, but for the state variables feedback exact linearization method was applied based on the exact state space model; the method was not robust to uncertainty of the parameters and models.

The nonlinear robust controller designed by adaptive backstepping was discussed in [3]. Although the internal and external disturbances of the system were considered, disturbance was just binding scaling, and the bound properties of the control variable were not considered. The nonlinear dispersion steam valve controller was designed by limiting the control variable for multimachine systems, which was difficult to achieve in [1] because of excessive computations. The adaptive backstepping method was used to design a nonlinear adaptive robust controller that simultaneously considered the input constraints of the main valve and the fast valve [4]. The steam valve controller was designed without considering the coordinated excitation and valve control. A design was 
proposed for a nonlinear adaptive robust controller based on adaptive backstepping in which coordinated excitation and valve control were simultaneously considered under input constraints [5]. However, disturbance was simply limited to the scaling of estimate bounds without consideration of state constraints. Although the state constraints of the main steam valve were considered, the impact of disruption perturbation was not considered in the design processes of [6-9].

Modern power systems are very complex, and they contain a wide variety of devices. These devices differ in physical properties and operating regions. Thus, the different constraints of these various devices should be considered in the automation of power systems [10]. Existing research results were obtained by common Lyapunov function, and the switching mechanism was introduced to obtain a better solution by establishing the constraints of control variable input. However, these studies did not indicate any limit to the state constraints for the system. The control problem for a class of constrained systems was solved by constructing a log type of symmetric Barrier Lyapunov Function [11]. A class of control problems with strictly limited output feedback nonlinear systems was studied in [12]. In the present paper, we propose a new type of log BLF (Barrier Lyapunov Function) to expand the range of the initial selection. This method is convenient and easy for the controller design process.

In addition, this paper introduces the system constraints of the status rotor running angle $\delta$ by constructing Barrier Lyapunov Function to establish the state bounds of the system. First, a nonlinear controller is designed by backstepping. Then, external disturbance is combined with Minimax to reduce the conservativeness of simple scaling disturbances. By using parameter-mapping mechanism, the adaptive law is designed by the upper and lower bounds of the parameters. Then, we estimate the uncertain parameters of the system and establish the coordinated excitation and valve control model. The switching mechanism is introduced to deal with the input amplitude constraint problems of the main steam and fast steam valve control in the system. To satisfy the system's state constraints, the controller is designed with backstepping which is nonlinear and simultaneously combined with relevant constrained signal and constraint values. The construction of a restrictive log type of Barrier Lyapunov Function guarantees that the state power angle set will be within the prescribed range. The Simulation results reveal that amplitude of the state response curve for the system is small, inhibitory effect of disturbance is good, and the limited control inputs are within the set range.

\section{Establishment of the Coordinated Excitation and Valve Control Model}

The dynamic equations of generator excitation winding are as follows:

$$
\begin{aligned}
& V_{f}=T_{d 0}^{\prime} \dot{E}_{q}^{\prime}+E_{q}-E_{f d s}, \\
& E_{q}=\frac{x_{d \Sigma}}{x_{d \Sigma}^{\prime}} E_{q}^{\prime}-\frac{\left(x_{d}-x_{d}^{\prime}\right) V_{s} \cos \delta}{x_{d \Sigma}^{\prime}},
\end{aligned}
$$

where $V_{f}$ is the excitation voltage, $T_{d 0}^{\prime}$ is the time constant of the excitation winding, $E_{q}^{\prime}$ is the $q$ axis transient voltage, $E_{q}$ is the no load electromotive force of generator, $E_{f d s}$ is given the excitation voltage of the stable operation, $V_{s}$ is the infinite bus voltage, $x_{d \Sigma}$ is the equivalent reactance between $E_{q}$ and $V_{s}, x_{d \Sigma}^{\prime}$ is the equivalent reactance between $E_{q}^{\prime}$ and $V_{s}$, and $x_{d}$ and $x_{d}^{\prime}$ are the $d$-axis reactance and transient reactance, respectively.

Thus, the excitation equation is

$$
\begin{aligned}
\dot{\delta}= & \omega-\omega_{0}, \\
\dot{\omega}= & -\frac{D}{H}\left(\omega-\omega_{0}\right)+\frac{\omega_{0}}{H}\left(P_{m}-\frac{E_{q}^{\prime} V_{s}}{x_{d \Sigma}^{\prime}} \sin \delta\right)+\xi_{1}, \\
\dot{E}_{q}^{\prime}= & \frac{1}{T_{d 0}^{\prime}}\left(-\frac{x_{d \Sigma}}{x_{d \Sigma}^{\prime}} E_{q}^{\prime}+\frac{x_{d}-x_{d}^{\prime}}{x_{d \Sigma}^{\prime}} V_{s} \cos \delta+E_{f d s}+V_{f}\right) \\
& +\xi_{2},
\end{aligned}
$$

where $\delta$ is the rotor angle of generator, $\omega$ is the rotor speed of the generator, $\omega_{0}$ is the generator synchronous speed, $H$ is the inertia of generator rotor, $D$ is the uncertain parameter of the system model, that is, damping coefficient which cannot be accurately measured, $\xi_{1}$ is external disturbance of the generator rotor, $\xi_{2}$ is external disturbance of the generator transient potential, and $\xi_{1}$ and $\xi_{2}$ are considered external disturbances of the system.

The model is based on the excitation control model of power system. The proposed model is relatively close to the actual model because of two factors for consideration. One is the uncertainty derived from the damping coefficient which is difficult to measure in the actual system. The second is the influence of external disturbances both on generator potential transient speed and on transient voltage.

Nowadays, middle reheat is widely used by large-capacity turbine generator sets, and its physical structure is shown in Figure 1.

The high-pressure cylinder and the middle-pressure cylinder are equivalent to an inertia link. The equivalent time constants are $T_{H L}$ and $T_{M L}$. The equivalent power distribution coefficients are $C_{H}$ and $C_{M L}$, with mechanical power output of $P_{H}$ and $P_{M L}$, respectively. The total mechanical power output of the prime mover is $P_{m}$, which is equal to the mechanical power output of the high-pressure cylinder and the middle-pressure cylinder expressed as $P_{H}$ and $P_{M L}$. Figure 2 shows the block diagram of the value control system transfer function.

From Figure 2, we can determine practical valve control system differential equations as follows:

$$
\begin{gathered}
\dot{P}_{H}=-\frac{1}{T_{H \Sigma}}\left(P_{H}-C_{H} P_{m 0}\right)+\frac{C_{H}}{T_{H \Sigma}} u_{1}+\xi_{3}, \\
u_{1 \text { min }} \leq u_{1} \leq u_{1 \text { max }}, \\
\dot{P}_{M L}=-\frac{1}{T_{M H \Sigma}}\left(P_{M L}-C_{M L} P_{m 0}\right)+\frac{C_{M L}}{T_{M L \Sigma}} u_{2}+\xi_{4}, \\
u_{2 \text { min }} \leq u_{2} \leq u_{2 \max },
\end{gathered}
$$




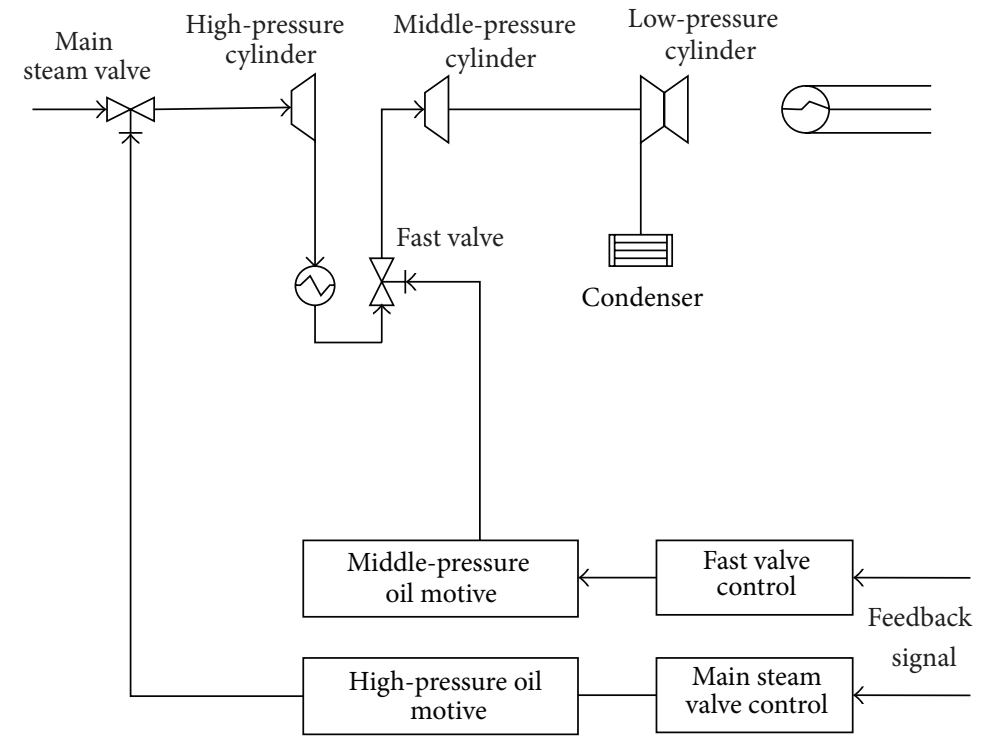

Figure 1: Physical structure model of reheat steam turbine.

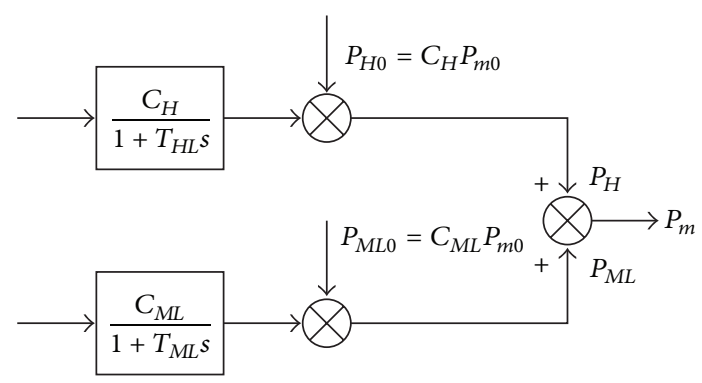

FIGURE 2: Block diagram of valve control system transfer function.

$$
\begin{gathered}
T_{H \Sigma}=T_{H}+T_{H g}, \\
T_{M L \Sigma}=T_{M L}+T_{M L g},
\end{gathered}
$$

where $T_{H}$ and $T_{H g}$ are the time constants of the high-pressure cylinder and the main valve oil motive, respectively; $T_{M L}$ and $T_{M L g}$ are the time constants of the low-pressure cylinder and the regulating valve oil motive, respectively; $P_{H}$ and $P_{M L}$ are the mechanical power outputs of the high-pressure cylinder and the middle-pressure cylinder, respectively; $T_{H \Sigma}$ is the sum time constant of the high-pressure cylinder and the main valve controller; $T_{M L \Sigma}$ is the sum time constant of the lowpressure cylinder and the fast valve controller; $C_{H}$ and $C_{M L}$ are the power distribution coefficients of the high-pressure cylinder and the low-pressure cylinder and are 0.7 and 0.3 , respectively; $u_{1}$ and $u_{2}$ are the control input of the main valve and the fast valve, respectively; $u_{1 \text { min }}$ and $u_{1 \text { max }}$ are the min and max control input of the main valve, respectively; $u_{2 \text { min }}$ and $u_{2 \max }$ are the min and max control input of the fast valve, respectively; $\xi_{3}$ and $\xi_{4}$ are the external disturbances of the main valve and the fast valve, respectively.

According to (3) and (4), we can obtain the system equations of the main valve and the fast valve as follows.
The main valve control equation is

$$
\begin{aligned}
& \dot{\delta}= \omega-\omega_{0}, \\
& \dot{\omega}=-\frac{D}{H}\left(\omega-\omega_{0}\right)+\frac{\omega_{0}}{H}\left(P_{H}+P_{M L}-\frac{E_{q}^{\prime} V_{s}}{X_{d \Sigma}^{\prime}} \sin \delta\right) \\
&+\xi_{1}, \\
& \dot{P}_{m}= \dot{P}_{H}=-\frac{1}{T_{H \Sigma}}\left(P_{H}-C_{H} P_{m 0}\right)+\frac{C_{H}}{T_{H \Sigma}} u_{1}+\xi_{3}, \\
& u_{1 \min } \leq u_{1} \leq u_{1 \max } .
\end{aligned}
$$

The fast valve control equation is

$$
\begin{aligned}
& \dot{\delta}= \omega-\omega_{0}, \\
& \dot{\omega}=-\frac{D}{H}\left(\omega-\omega_{0}\right)+\frac{\omega_{0}}{H}\left(P_{H}+P_{M L}-\frac{E_{q}^{\prime} V_{s}}{X_{d \Sigma}^{\prime}} \sin \delta\right) \\
&+\xi_{1}, \\
& \dot{P}_{m}= \dot{P}_{M L}=-\frac{1}{T_{M L \Sigma}}\left(P_{M L}-C_{M L} P_{m 0}\right)+\frac{C_{M L}}{T_{M L \Sigma}} u_{2}+\xi_{4}, \\
& u_{2 \min } \leq u_{2} \leq u_{2 \max } .
\end{aligned}
$$

The control model of the main valve and the fast valve contains the uncertain parameters $D$, external disturbances $\xi_{1}, \xi_{2}$, and $\xi_{3}$, and input constraint bounds of the main valve and the fast valve control. 
In summary, the excitation and the main valve equations are as follows:

$$
\begin{aligned}
\dot{\delta}= & \omega-\omega_{0}, \\
\dot{\omega}= & -\frac{D}{H}\left(\omega-\omega_{0}\right)+\frac{\omega_{0}}{H}\left(P_{m}-\frac{E_{q}^{\prime} V_{s}}{x_{d \Sigma}^{\prime}} \sin \delta\right)+\xi_{1}, \\
\dot{E}_{q}^{\prime}= & \frac{1}{T_{d 0}^{\prime}}\left(-\frac{x_{d \Sigma}}{x_{d \Sigma}^{\prime}} E_{q}^{\prime}+\frac{x_{d}-x_{d}^{\prime}}{x_{d \Sigma}^{\prime}} V_{s} \cos \delta+E_{f d s}+u_{1}\right) \\
& +\xi_{2}, \\
\dot{P}_{m}= & \dot{P}_{H}=-\frac{1}{T_{H \Sigma}}\left(P_{H}-C_{H} P_{m 0}\right)+\frac{C_{H}}{T_{H \Sigma}} u_{2}+\xi_{3} .
\end{aligned}
$$

The excitation and the fast valve equations are as follows:

$$
\begin{aligned}
\dot{\delta}= & \omega-\omega_{0}, \\
\dot{\omega}= & -\frac{D}{H}\left(\omega-\omega_{0}\right)+\frac{\omega_{0}}{H}\left(P_{m}-\frac{E_{q}^{\prime} V_{s}}{x_{d \Sigma}^{\prime}} \sin \delta\right)+\xi_{1}, \\
\dot{E}_{q}^{\prime}= & \frac{1}{T_{d 0}^{\prime}}\left(-\frac{x_{d \Sigma}}{x_{d \Sigma}^{\prime}} E_{q}^{\prime}+\frac{x_{d}-x_{d}^{\prime}}{x_{d \Sigma}^{\prime}} V_{s} \cos \delta+E_{f d s}+u_{1}\right) \\
& +\xi_{2}, \\
\dot{P}_{m}= & \dot{P}_{M L}=-\frac{1}{T_{M H \Sigma}}\left(P_{M L}-C_{M L} P_{m 0}\right)+\frac{C_{M L}}{T_{M L \Sigma}} u_{2}+\xi_{4} .
\end{aligned}
$$

The two switching subsystems are the excitation and main valve control system and the excitation and fast valve control system. These systems are very similar and therefore we can only choose coordinated control of the excitation and main valve for instructing the controller design process.

The coordinated control model of the excitation and main valve is as follows:

$$
\begin{aligned}
\dot{x}_{1}= & x_{2}, \quad-k_{b}<x_{1}<k_{b}, \\
\dot{x}_{2}= & \theta x_{2}+k_{1} x_{4}+k_{1} x_{5}+a_{0}+k_{2} \sin \left(x_{1}+\delta_{0}\right) x_{3} \\
& +k_{9} \sin \left(x_{1}+\delta_{0}\right)+\xi_{1}, \\
\dot{x}_{3}= & k_{3} x_{3}+k_{5}+k_{4} \cos \left(x_{1}+\delta_{0}\right)+k_{6} u_{1}+\xi_{2}, \\
\dot{x}_{4}= & k_{7} x_{4}+k_{8} u_{2}+\xi_{3}, \\
\dot{x}_{5}= & 0, \\
z= & {\left[\begin{array}{ll}
q_{1} x_{1} & q_{2} x_{2}
\end{array}\right]^{T}, }
\end{aligned}
$$

where $k_{b}$ and $-k_{b}$ are the upper and lower bounds of $x_{1}$, respectively, $-D / H=\theta, \omega_{0} / H=k_{1},\left(\omega_{0} / H\right) P_{m 0}=a_{0}$, $-\omega_{0} V_{s} / H x_{d \Sigma}^{\prime}=k_{2},-x_{d \Sigma} / T_{d 0}^{\prime} x_{d \Sigma}^{\prime}=k_{3},\left(\left(x_{d}-x_{d}^{\prime}\right) / T_{d 0}^{\prime} x_{d \Sigma}^{\prime}\right) V_{s}=$ $k_{4},-x_{d \Sigma} E_{q 0}^{\prime} / T_{d 0}^{\prime} x_{d \Sigma}^{\prime}+E_{f d s} / T_{d 0}^{\prime}=k_{5}, 1 / T_{d 0}^{\prime}=k_{6},-1 / T_{H \Sigma}=$ $k_{7}, C_{H} / T_{H \Sigma}=k_{8},-\omega_{0} E_{q}^{\prime} V_{s} /\left(1+X_{d \Sigma}^{\prime}\right)=k_{9}, z$ is the regulating output, $q_{1}$ and $q_{2}$ are nonnegative weighting coefficients that account for the weighted proportion between $x_{1}$ and $x_{2}$, with $q_{1}^{2}+q_{2}^{2} \leq 1, D$ is the uncertain parameter that is difficult to accurately measure, and $\theta$ is an uncertain constant.

\section{Excitation and Valve Controller Design of State and Input Constraints}

Lemma 1 (see [12]). A Barrier Lyapunov Function is a scalar function $V(x)$, defined with respect to the system $\dot{x}=f(x)$ on an open region $D$ containing the origin, that is continuous, positive definite, has continuous first-order partial derivatives at every point of $D$, has the property $V(x) \rightarrow \infty$ as $x$ approaches the boundary of $D$, and satisfies $V(x) \leq b \forall t>0$ along the solution of $\dot{x}=f(x)$ for $x(0) \in D$ and some positive constant $b$.

Lemma 2 (see [12]). For any positive constants $k_{b}$, let $Z_{1}:=$ $\left\{z_{1} \in R:-k_{b}<z_{1}<k_{b}\right\} \subset R$ and $w \subset R^{l-1}$ be open sets. Consider the system $\dot{\mu}=h(t, \mu)$, where $\mu=\left[z_{1}, w\right]^{T}$ and $h \subset R^{l}$ is piecewise continuous in $t$. Suppose that there exist functions $U: w \rightarrow R_{+}$and $V_{1}: Z_{1} \rightarrow R_{+}$, continuously differentiable and positive definite in their respective domains, such that

$$
\begin{aligned}
& V_{1}\left(z_{1}\right) \longrightarrow \infty \text { as } z_{1} \longrightarrow-k_{b} \text { or } z_{1} \longrightarrow k_{b}, \\
& \lambda_{1}(\|w\|) \leq U(w) \leq \lambda_{2}(\|w\|),
\end{aligned}
$$

where $\lambda_{1}$ and $\lambda_{2}$ are class $K_{\infty}$ functions. Let $V(\mu)=V_{1}\left(z_{1}\right)+$ $U(w)$, and $z_{1}(0)$ belong to the set $Z_{1}$. If the inequality holds $\dot{V}=(\partial V / \partial \mu) h \leq 0$, then $z_{1}(t)$ remains in the open set $Z_{1}$.

Step 1. Considering $x_{2}$ to be a virtual controller and choosing $x_{2}^{*}=-c_{1}\left(k_{b}^{2}-e_{1}^{2}\right) e_{1}, c_{1}>0$, let $e_{1}=x_{1}, e_{2}=x_{2}-x_{2}^{*}$; we obtain the common Barrier Lyapunov Function of $V_{1}$ in the first step as follows:

$$
V_{1}=\frac{1}{2} \log \frac{k_{b}^{2}}{k_{b}^{2}-e_{1}^{2}} .
$$

Then

$$
\dot{V}_{1}=\frac{1}{2} \frac{k_{b}^{2}-e_{1}^{2}}{k_{b}^{2}} \cdot \frac{2 e_{1} k_{b}^{2} \dot{e}_{1}}{\left(k_{b}^{2}-e_{1}^{2}\right)^{2}}=\frac{e_{1} e_{2}}{k_{b}^{2}-e_{1}^{2}}-c_{1} e_{1}^{2} .
$$

Clearly, if $e_{2}=0$, we have $\dot{V}_{1}<0$.

Step 2. We consider the second equation of (10), our task in this step is to make $e_{2} \rightarrow 0$. By augmenting $V_{1}$, the Lyapunov function $V_{2}$ is choosen as $V_{2}=V_{1}+(1 / 2) e_{2}^{2}$. Satisfying dissipation inequality for arbitrary disturbance, we define the energy function as

$$
H_{1}=\dot{V}_{2}+\frac{1}{2}\left(\|z\|^{2}-\gamma^{2}\left\|\xi_{1}\right\|^{2}\right) .
$$

The performance function is

$$
J_{1}=\int_{0}^{\infty}\left(\|z\|^{2}-\gamma^{2}\left\|\xi_{1}\right\|^{2}\right) d t
$$

because

$$
\begin{aligned}
\dot{V}_{2} & =\frac{e_{1} e_{2}}{k_{b}^{2}-e_{1}^{2}}-c_{1} e_{1}^{2}+e_{2}\left(\theta x_{2}+k_{1} z_{4}+a_{0}\right. \\
& +k_{2} \sin \left(x_{1}+\delta_{0}\right) x_{3}+k_{9} \sin \left(x_{1}+\delta_{0}\right)+\xi_{1} \\
& \left.+c_{1} k_{b}^{2} x_{2}-3 c_{1} e_{1}^{2} x_{2}\right) .
\end{aligned}
$$


By substituting (16) into (14), we get

$$
\begin{aligned}
H_{1} & =-c_{1} e_{1}^{2}+e_{2}\left(\frac{e_{1}}{k_{b}^{2}-e_{1}^{2}}+\theta x_{2}+k_{1} z_{4}+a_{0}\right. \\
& +k_{2} \sin \left(x_{1}+\delta_{0}\right) x_{3}+k_{9} \sin \left(x_{1}+\delta_{0}\right)+\xi_{1} \\
& \left.+c_{1} k_{b}^{2} x_{2}-3 c_{1} e_{1}^{2} x_{2}\right)+\frac{1}{2} q_{1}^{2} x_{1}^{2}+\frac{1}{2} q_{2}^{2} x_{2}^{2}-\frac{1}{2} \gamma^{2} \xi_{1}^{2} .
\end{aligned}
$$

Take the first partial derivative of $H_{1}$ for $\xi_{1}$ and let $\dot{H}_{1}=0$; then $\partial H_{1} / \partial \xi_{1}=e_{2}-\gamma^{2} \xi_{1}=0$. Therefore, we can obtain

$$
\xi_{1}^{*}=\frac{e_{2}}{\gamma^{2}}
$$

Take the second partial derivative of $H_{1}$ for $\xi_{1}$; then we get $\partial^{2} H_{1} / \partial \xi_{1}^{2}=-(1 / 2) \gamma^{2}<0$. We know that $H_{1}$ has a maximum value for $\xi_{1}$. According to the proof presented in the previous chapter, we know that $H_{1}$ can get the maximum for $\xi_{1}^{*}$. Thus, we can conclude that the performance $J_{1}$ reaches the maximum. Clearly, $\xi_{1}^{*}$ is the greatest degree of disturbance impact on the system.

By substituting (18) into (17), we get

$$
\begin{aligned}
H_{1} & =-\alpha e_{1}^{2}+e_{2}\left(\frac{e_{1}}{k_{b}^{2}-e_{1}^{2}}+\theta x_{2}+k_{1} z_{4}+a_{0}\right. \\
& +k_{2} \sin \left(x_{1}+\delta_{0}\right) x_{3}+k_{9} \sin \left(x_{1}+\delta_{0}\right)+h_{1} x_{2} \\
& \left.-3 c_{1} e_{1}^{2} x_{2}+h_{2} e_{2}-q_{2}^{2} c_{1}\left(k_{b}^{2}-e_{1}^{2}\right) e_{1}\right),
\end{aligned}
$$

where $\alpha=c_{1}-(1 / 2) q_{1}^{2}-(1 / 2) q_{2}^{2} c_{1}^{2}\left(k_{b}^{2}-e_{1}^{2}\right)^{2}, h_{1}=c_{1} k_{b}^{2}$, and $h_{2}=1 / 2 \gamma^{2}+(1 / 2) q_{2}^{2}$.

Let

$$
\begin{aligned}
& h_{2} e_{2}+k_{2} \sin \left(x_{1}+\delta_{0}\right) x_{3}+k_{9} \sin \left(x_{1}+\delta_{0}\right)=-e_{2}, \\
& \frac{e_{1}}{k_{b}^{2}-e_{1}^{2}}+a_{0}+\theta x_{2}+k_{1} z_{4}+h_{1} x_{2}-q_{2}^{2} c_{1}\left(k_{b}^{2}-e_{1}^{2}\right) e_{1} \\
& \quad-3 c_{1} e_{1}^{2} x_{2}=-e_{2} .
\end{aligned}
$$

We can get the virtual control

$$
\begin{aligned}
x_{3}^{*} & =-\frac{1}{k_{2} \sin \left(x_{1}+\delta_{0}\right)}\left(h_{3} e_{2}+k_{9} \sin \left(x_{1}+\delta_{0}\right)\right), \\
z_{4}^{*} & =-\frac{1}{k_{1}}\left(\frac{e_{1}}{k_{b}^{2}-e_{1}^{2}}+a_{0}+\widehat{\theta} x_{2}+h_{1} x_{2}\right. \\
& \left.-q_{2}^{2} c_{1}\left(k_{b}^{2}-e_{1}^{2}\right) e_{1}-3 c_{1} e_{1}^{2} x_{2}+e_{2}\right),
\end{aligned}
$$

where $h_{3}=h_{2}+1$, and we use the estimated $\hat{\theta}$ as $\theta$. Then

$$
H_{1}=-\alpha e_{1}^{2}-2 e_{2}^{2}+(\theta-\widehat{\theta}) x_{2} e_{2} .
$$

Step 3. Considering the unknown parameter $\theta$ in the second equation of $(10), \theta \in\left[\theta_{\min } \theta_{\max }\right] ; \theta_{\min }$, and $\theta_{\max }$ are, respectively, the upper and lower bounds of $\theta$. Augmented $V_{2}$, the function of a common Lyapunov function of subsystem 1 and subsystem 2 , is

$$
V_{3}=V_{2}+\frac{1}{2} e_{3}^{2}+\frac{1}{2} e_{4}^{2}+\frac{1}{2 \rho}\left[(\bar{\theta}-\theta)^{2}-(\bar{\theta}-\widehat{\theta})^{2}\right],
$$

where $\bar{\theta}$ is a mapping mechanism, $\hat{\theta}$ is the estimate of $\theta, \rho$ is the adaptive law of gain, $\rho>0, e_{3}=x_{3}-x_{3}^{*}$, and $e_{4}=z_{4}-z_{4}^{*}$.

We select the energy function

$$
H_{2}=\dot{V}_{3}+\frac{1}{2}\left(\|z\|^{2}-\gamma^{2}\|\xi\|^{2}\right)
$$

The performance function is

$$
J_{2}=\int_{0}^{\infty}\left(\|z\|^{2}-\gamma^{2}\|\xi\|^{2}\right) d t
$$

where $\xi=\left[\begin{array}{lll}\xi_{1} & \xi_{2} & \xi_{3}\end{array}\right]^{T}$ and the derivative of formula (23) is

$$
\dot{V}_{3}=\dot{V}_{2}+e_{3} \dot{e}_{3}+\frac{1}{\rho}[(\bar{\theta}-\theta) \dot{\bar{\theta}}-(\bar{\theta}-\widehat{\theta})(\dot{\bar{\theta}}-\dot{\hat{\theta}})]
$$

The derivatives of formula (21) are

$$
\begin{aligned}
& \dot{x}_{3}^{*}=-\frac{h_{3}\left(\theta x_{2}+k_{1} z_{4}+a_{0}+k_{2} \sin \left(x_{1}+\delta_{0}\right) x_{3}+k_{9} \sin \left(x_{1}+\delta_{0}\right)+e_{2} / \gamma^{2}\right)}{k_{2} \sin \left(x_{1}+\delta_{0}\right)} \\
&-\frac{h_{3}\left(c_{1} k_{b}^{2} x_{2}-3 c_{1} e_{1}^{2} x_{2}\right)+k_{9} \cos \left(x_{1}+\delta_{0}\right) x_{2}}{k_{2} \sin \left(x_{1}+\delta_{0}\right)}-\frac{\left(h_{3} e_{2}+k_{9} \sin \left(x_{1}+\delta_{0}\right)\right) k_{2} \cos \left(x_{1}+\delta_{0}\right) x_{2}}{k_{2}^{2} \sin ^{2}\left(x_{1}+\delta_{0}\right)}, \\
& \dot{z}_{4}^{*}=-\frac{1}{k_{1}}\left(\frac{x_{2}\left(k_{b}^{2}+e_{1}^{2}\right)}{\left(k_{b}^{2}-e_{1}^{2}\right)^{2}}+\dot{\hat{\theta}} x_{2}+\widehat{\theta} \dot{x}_{2}+\left(h_{1}+1\right) \dot{x}_{2}+\left(3 q_{2}^{2} c_{1}-3 c_{1}\right) e_{1}^{2} x_{2}-6 c_{1} e_{1} x_{2}^{2}-3 c_{1} e_{1}^{2} \dot{x}_{2}+\left(c_{1} k_{b}^{2}-q_{2}^{2} c_{1} k_{b}^{2}\right) x_{2}\right) .
\end{aligned}
$$


By substituting from (26) to (28) into (24), we get

$$
\begin{aligned}
H_{2} & =H_{1}+e_{3}\left(k_{3} x_{3}+k_{5}+k_{4} \cos \left(x_{1}+\delta_{0}\right)+k_{6} u_{1}\right. \\
& \left.+\xi_{2}-\dot{x}_{3}\right)+e_{4}\left(k_{7} x_{4}+k_{8} u_{2}+\xi_{3}-\dot{z}_{4}\right)-\frac{1}{2} \gamma^{2} \xi_{2}^{2} \\
& -\frac{1}{2} \gamma^{2} \xi_{3}^{2}-\frac{1}{\rho}((\theta-\hat{\theta}) \dot{\bar{\theta}}-(\bar{\theta}-\hat{\theta}) \dot{\hat{\theta}}) .
\end{aligned}
$$

Take the first partial derivative of $H_{2}$ for $\xi_{2}$ and $\xi_{3}$ and make them equal to 0 ; then

$$
\begin{aligned}
& \frac{\partial H_{2}}{\partial \xi_{2}}=e_{3}-\gamma^{2} \xi_{2}=0, \\
& \frac{\partial H_{2}}{\partial \xi_{3}}=e_{4}-\gamma^{2} \xi_{3}=0 .
\end{aligned}
$$

Therefore

$$
\begin{aligned}
& \xi_{2}^{*}=\frac{e_{3}}{\gamma^{2}}, \\
& \xi_{3}^{*}=\frac{e_{4}}{\gamma^{2}} .
\end{aligned}
$$

Take the second partial derivative of $\mathrm{H}_{2}$ for $\xi_{2}$ and $\xi_{3}$; then we can get $\partial^{2} H_{2} / \partial \xi_{2}^{2}=-\gamma^{2}<0$ and $\partial^{2} H_{2} / \partial \xi_{3}^{2}=-\gamma^{2}<0$. We know that $\mathrm{H}_{2}$ has a respective maximum value for $\xi_{2}$ and $\xi_{3}$. Therefore, because $\xi_{2}^{*}$ and $\xi_{3}^{*}$ can make $H_{2}$ reach the maximum value, we can also make the performance $J_{2}$ reach the maximum. Clearly, $\xi_{2}^{*}$ and $\xi_{3}^{*}$ are really the greatest degrees of disturbance impact on the system.

By substituting (31) into (29), we get

$$
\begin{aligned}
H_{2} & =H_{1}+e_{3}\left(k_{3} x_{3}+k_{5}+k_{4} \cos \left(x_{1}+\delta_{0}\right)+k_{6} u_{1}+\frac{e_{3}}{2 \gamma^{2}}\right. \\
& +\frac{h_{3}\left(\theta x_{2}+k_{1} z_{4}+a_{0}+k_{2} \sin \left(x_{1}+\delta_{0}\right) x_{3}+k_{9} \sin \left(x_{1}+\delta_{0}\right)+e_{2} / \gamma^{2}\right)}{k_{2} \sin \left(x_{1}+\delta_{0}\right)}+\frac{h_{3}\left(c_{1} k_{b}^{2} x_{2}-3 c_{1} e_{1}^{2} x_{2}\right)+k_{9} \cos \left(x_{1}+\delta_{0}\right) x_{2}}{k_{2} \sin \left(x_{1}+\delta_{0}\right)} \\
& \left.+\frac{\left(h_{3} e_{2}+k_{9} \sin \left(x_{1}+\delta_{0}\right)\right) k_{2} \cos \left(x_{1}+\delta_{0}\right) x_{2}}{k_{2}^{2} \sin ^{2}\left(x_{1}+\delta_{0}\right)}\right)+e_{4}\left(k_{7} x_{4}+k_{8} u_{2}+\frac{e_{4}}{2 \gamma^{2}}\right. \\
& \left.+\frac{1}{k_{1}}\left(\frac{x_{2}\left(k_{b}^{2}+e_{1}^{2}\right)}{\left(k_{b}^{2}-e_{1}^{2}\right)^{2}}+\dot{\hat{\theta}} x_{2}+\hat{\theta} \dot{x}_{2}+\left(h_{1}+1\right) \dot{x}_{2}+\left(3 q_{2}^{2} c_{1}-3 c_{1}\right) e_{1}^{2} x_{2}-6 c_{1} e_{1} x_{2}^{2}-3 c_{1} e_{1}^{2} \dot{x}_{2}+\left(c_{1} k_{b}^{2}-q_{2}^{2} c_{1} k_{b}^{2}\right) x_{2}\right)\right) \\
& -\frac{1}{\rho}((\theta-\hat{\theta}) \dot{\bar{\theta}}-(\bar{\theta}-\hat{\theta}) \dot{\hat{\theta}}) .
\end{aligned}
$$

Let

$$
\begin{aligned}
-c_{3} e_{3}= & k_{3} x_{3}+k_{5}+k_{4} \cos \left(x_{1}+\delta_{0}\right)+k_{6} u_{1}+x_{3}+\frac{e_{3}}{2 \gamma^{2}}+\frac{k_{9} \sin \left(x_{1}+\delta_{0}\right)+e_{2} / \gamma^{2}}{k_{2} \sin \left(x_{1}+\delta_{0}\right)} \\
& +\frac{h_{3}\left(c_{1} k_{b}^{2} x_{2}-3 c_{1} e_{1}^{2} x_{2}\right)+k_{9} \cos \left(x_{1}+\delta_{0}\right) x_{2}+h_{3}\left(\theta x_{2}+k_{1} z_{4}+a_{0}\right) x_{3}}{k_{2} \sin \left(x_{1}+\delta_{0}\right)} \\
& +\frac{\left(h_{3} e_{2}+k_{9} \sin \left(x_{1}+\delta_{0}\right)\right) k_{2} \cos \left(x_{1}+\delta_{0}\right) x_{2}}{k_{2}^{2} \sin ^{2}\left(x_{1}+\delta_{0}\right)}, \\
-c_{3} e_{4}= & k_{7} x_{4}+k_{8} u_{2}+\frac{e_{4}}{2 \gamma^{2}} \\
& +\frac{1}{k_{1}}\left(\frac{x_{2}\left(k_{b}^{2}+e_{1}^{2}\right)}{\left(k_{b}^{2}-e_{1}^{2}\right)^{2}}+\dot{\hat{\theta}} x_{2}+\hat{\theta} \dot{x}_{2}+\left(h_{1}+1\right) \dot{x}_{2}+\left(3 q_{2}^{2} c_{1}-3 c_{1}\right) e_{1}^{2} x_{2}-6 c_{1} e_{1} x_{2}^{2}-3 c_{1} e_{1}^{2} \dot{x}_{2}+\left(c_{1} k_{b}^{2}-q_{2}^{2} c_{1} k_{b}^{2}\right) x_{2}\right) .
\end{aligned}
$$


The controller functions are

$$
\begin{aligned}
u_{1} & =-\frac{1}{k_{6}}\left(k_{3} x_{3}+k_{5}+k_{4} \cos \left(x_{1}+\delta_{0}\right)+\frac{e_{3}}{2 \gamma^{2}}+c_{3} e_{3}\right. \\
& +\frac{h_{3}\left(\widehat{\theta} x_{2}+k_{1} z_{4}+a_{0}+k_{2} \sin \left(x_{1}+\delta_{0}\right) x_{3}+k_{9} \sin \left(x_{1}+\delta_{0}\right)+e_{2} / \gamma^{2}\right)}{k_{2} \sin \left(x_{1}+\delta_{0}\right)}+\frac{h_{3}\left(c_{1} k_{b}^{2} x_{2}-3 c_{1} e_{1}^{2} x_{2}\right)+k_{9} \cos \left(x_{1}+\delta_{0}\right) x_{2}}{k_{2} \sin \left(x_{1}+\delta_{0}\right)} \\
& \left.+\frac{\left(h_{3} e_{2}+k_{9} \sin \left(x_{1}+\delta_{0}\right)\right) k_{2} \cos \left(x_{1}+\delta_{0}\right) x_{2}}{k_{2}^{2} \sin ^{2}\left(x_{1}+\delta_{0}\right)}\right), \\
u_{2} & =-\frac{1}{k_{8}}\left(k_{7} x_{4}+\frac{e_{4}}{2 \gamma^{2}}+c_{4} e_{4}+\frac{1}{k_{1}}\left(\frac{x_{2}\left(k_{b}^{2}+e_{1}^{2}\right)}{\left(k_{b}^{2}-e_{1}^{2}\right)^{2}}+\dot{\hat{\theta}} x_{2}+\hat{\theta} \dot{x}_{2}\right.\right. \\
& +\left(h_{1}+1-3 c_{1} e_{1}^{2}\right)\left(\hat{\theta} x_{2}+k_{1} z_{4}+a_{0}+k_{2} \sin \left(x_{1}+\delta_{0}\right) x_{3}+k_{9} \sin \left(x_{1}+\delta_{0}\right)+\frac{e_{2}}{\gamma^{2}}\right)+\left(3 q_{2}^{2} c_{1}-3 c_{1}\right) e_{1}^{2} x_{2}-6 c_{1} e_{1} x_{2}^{2} \\
& \left.\left.+\left(c_{1} k_{b}^{2}-q_{2}^{2} c_{1} k_{b}^{2}\right) x_{2}\right)\right) .
\end{aligned}
$$

By substituting (34) into (32), we get

$$
\begin{aligned}
H_{2}= & -\alpha e_{1}^{2}-2 e_{2}^{2}-c_{2} e_{3}^{2}-c_{3} e_{4}^{2}+(\theta-\widehat{\theta}) x_{2} e_{2} \\
& +\frac{e_{4}}{k_{1}}\left(\widehat{\theta}-3 c_{1} e_{1}^{2}\right)(\theta-\widehat{\theta}) x_{2} \\
& +\frac{1}{k_{1}}\left(h_{1}+1\right)(\theta-\widehat{\theta}) e_{4} x_{2}+\frac{h_{3}(\theta-\hat{\theta}) x_{2}}{k_{2} \sin \left(x_{1}+\delta_{0}\right)} e_{3} \\
& -\frac{1}{\rho}[(\theta-\hat{\theta}) \dot{\bar{\theta}}-(\bar{\theta}-\hat{\theta}) \dot{\hat{\theta}}] .
\end{aligned}
$$

The adaptive law follows

$$
\begin{aligned}
\dot{\bar{\theta}} & =\rho\left(x_{2} e_{2}+\frac{h_{3} x_{2}}{k_{2} \sin \left(x_{1}+\delta_{0}\right)} e_{3}+\frac{e_{4}}{k_{1}}\left(\widehat{\theta}-3 c_{1} e_{1}^{2}\right) x_{2}\right. \\
& \left.+\frac{1}{k_{1}}\left(h_{1}+1\right) e_{4} x_{2}-\frac{\dot{\hat{\theta}}}{\rho(\theta-\hat{\theta})}(\bar{\theta}-\hat{\theta})\right),
\end{aligned}
$$

where

$$
\widehat{\theta}= \begin{cases}\bar{\theta}, & \theta_{\min } \leq \bar{\theta} \leq \theta_{\max }, \\ \theta_{\min }, & \bar{\theta}<\theta_{\min }, \\ \theta_{\max }, & \bar{\theta}>\theta_{\max } .\end{cases}
$$

Then, we can obtain

$$
H_{2}=-\alpha e_{1}^{2}-2 e_{2}^{2}-c_{2} e_{3}^{2}-c_{3} e_{4}^{2}<0 .
$$

Let $V=2 V_{3}$; we get $\dot{V}=2 \dot{V}_{3} \leq\left(\gamma^{2}\|\xi\|^{2}-\|z\|^{2}\right)$, taking integral from both sides as follows:

$$
V(\infty)-V(0) \leq \int_{0}^{\infty}\left(\gamma^{2}\|\xi\|^{2}-\|z\|^{2}\right) .
$$

Satisfying the dissipation inequality, the closed-loop system (10) is stable asymptotically.

To avoid duplication, we can design coordinated excitation and valve control and just provide the design results as follows:

$$
\begin{aligned}
u_{1} & =-\frac{1}{k_{6}}\left(k_{3} x_{3}+k_{5}+k_{4} \cos \left(x_{1}+\delta_{0}\right)+\frac{e_{3}}{2 \gamma^{2}}+c_{3} e_{3}\right. \\
& +\frac{h_{3}\left(\widehat{\theta} x_{2}+k_{1} z_{4}+a_{0}+k_{2} \sin \left(x_{1}+\delta_{0}\right) x_{3}+k_{9} \sin \left(x_{1}+\delta_{0}\right)+e_{2} / \gamma^{2}\right)}{k_{2} \sin \left(x_{1}+\delta_{0}\right)}+\frac{h_{3}\left(c_{1} k_{b}^{2} x_{2}-3 c_{1} e_{1}^{2} x_{2}\right)+k_{9} \cos \left(x_{1}+\delta_{0}\right) x_{2}}{k_{2} \sin \left(x_{1}+\delta_{0}\right)} \\
& \left.+\frac{\left(h_{3} e_{2}+k_{9} \sin \left(x_{1}+\delta_{0}\right)\right) k_{2} \cos \left(x_{1}+\delta_{0}\right) x_{2}}{k_{2}^{2} \sin ^{2}\left(x_{1}+\delta_{0}\right)}\right),
\end{aligned}
$$




$$
\begin{aligned}
u_{3} & =-\frac{1}{k_{11}}\left(k_{10} x_{5}+\frac{e_{4}}{2 \gamma^{2}}+c_{4} e_{4}+\frac{1}{k_{1}}\left(\frac{x_{2}\left(k_{b}^{2}+e_{1}^{2}\right)}{\left(k_{b}^{2}-e_{1}^{2}\right)^{2}}+\dot{\hat{\theta}} x_{2}+\widehat{\theta} \dot{x}_{2}\right.\right. \\
& +\left(h_{1}+1-3 c_{1} e_{1}^{2}\right)\left(\widehat{\theta} x_{2}+k_{1} z_{4}+a_{0}+k_{2} \sin \left(x_{1}+\delta_{0}\right) x_{3}+k_{9} \sin \left(x_{1}+\delta_{0}\right)+\frac{e_{2}}{\gamma^{2}}\right)+\left(3 q_{2}^{2} c_{1}-3 c_{1}\right) e_{1}^{2} x_{2}-6 c_{1} e_{1} x_{2}^{2} \\
& \left.\left.+\left(c_{1} k_{b}^{2}-q_{2}^{2} c_{1} k_{b}^{2}\right) x_{2}\right)\right),
\end{aligned}
$$

where $k_{10}=-1 / T_{M L \Sigma}$ and $k_{11}=C_{M L} / T_{M L \Sigma}$. We design the adaptive law as follows

$$
\begin{aligned}
\dot{\bar{\theta}} & =\rho\left(x_{2} e_{2}+\frac{h_{3} x_{2}}{k_{2} \sin \left(x_{1}+\delta_{0}\right)} e_{3}+\frac{e_{4}}{k_{1}}\left(\hat{\theta}-3 c_{1} e_{1}^{2}\right) x_{2}\right. \\
& \left.+\frac{1}{k_{1}}\left(h_{1}+1\right) e_{4} x_{2}-\frac{\dot{\hat{\theta}}}{\rho(\theta-\hat{\theta})}(\bar{\theta}-\hat{\theta})\right),
\end{aligned}
$$

where

$$
\widehat{\theta}= \begin{cases}\bar{\theta}, & \theta_{\min } \leq \bar{\theta} \leq \theta_{\max }, \\ \theta_{\min }, & \bar{\theta}<\theta_{\min }, \\ \theta_{\max }, & \bar{\theta}>\theta_{\max } .\end{cases}
$$

Theorem 3. For the closed-loop system (10), under the condition of Lemma 1, satisfying the initial condition as follows $x_{1}(0) \in \Omega_{x 10}=\left\{x_{1}(0) \in R^{3}\right.$ and $\left.\left|x_{1}(0)\right|<k_{b}\right\}$, the error variable $x_{1}$ is always in the compact set $\Omega_{x 1}$ as follows:

$$
\Omega_{x 1}=\left\{x_{1} \in R^{3}:\left|x_{1}\right| \leq k_{b} \sqrt{1-e^{-2 V_{3}(0)}}\right\} \text {. }
$$

Proof. Due to $\dot{V}_{3} \leq 0$, we get $V_{3}(t) \leq V_{3}(0)$. For $\left|x_{1}(0)\right|<$ $k_{b}$, the state variable $x_{1}(t)$ satisfies $\left|x_{1}(t)\right|<k_{b}$, acoording to Lemma 2. Because of the inequality $(1 / 2) \log \left(k_{b}^{2} /\left(k_{b}^{2}-x_{1}^{2}\right)\right) \leq$ $V_{3}(t) \leq V_{3}(0)$, we can get $\left|x_{1}\right| \leq k_{b} \sqrt{1-e^{-2 V_{3}(0)}}$.

\section{Design of Switching Law}

The switching law is designed to ensure that the amplitude controller constraints are not destroyed in this section. Given the existence of a common Lyapunov function in subsystems, subsystems 1 and 2 are stable, and switching systems are stable under arbitrary switching laws. Thus, switching law can be designed in any form to avoid frequent switching systems. Thus, we design the hysteresis switching law as follows [13].

Hysteresis Switching Law. Consider $\sigma(0)=1$, for $t>0$; if $\sigma\left(t^{-}\right)=i \in\{1,2\}, u_{i \text { min }} \leq u_{i} \leq u_{i \text { max }}$, then holding $\sigma(t)=i$. If $\sigma\left(t^{-}\right)=1$, but $u_{1}<u_{1 \text { min }}$ or $u_{1}>u_{1 \max }$, then we get $\sigma(t)=2$; similarly, if $\sigma\left(t^{-}\right)=2$, but $u_{2}<$ $u_{2 \min }$ or $u_{2}>u_{2 \max }$, then we obtain $\sigma(t)=1$, where $\sigma(t)$ is the switching signal $\sigma(t)=1$, showing that system runs subsystem 1 , the main valve control system; consider $\sigma(t)=2$, showing that system runs subsystem 2 , the fast valve control system. $u_{i \text { min }}$ and $u_{i \max }$ are, respectively, the opening minimum and maximum of the main valve or the quick steam valve. The hysteresis switching law ensures that the system switches subsystem 2, after the system runs for some time, even if the control input satisfies subsystem 1, to avoid frequent switching and continue running subsystem 2 , until the constraints are reached.

\section{Simulation Analysis}

The system parameters are selected as $V_{s}=1, T_{H \Sigma}=0.2$, $T_{M L \Sigma}=0.35, C_{H}=0.3, C_{M L}=0.7, H=10, u_{1 \min }=u_{2 \min }=$ -3 , and $u_{1 \max }=u_{2 \max }=6$. We choose the stable operation points of the system as $\delta_{0}=57.3^{\circ}, \omega_{0}=314.159 \mathrm{rad} / \mathrm{s}, P_{m 0}=$ $0.852, E_{q 0}^{\prime}=0.3913, u_{1 \min }=u_{2 \min }=-4, u_{1 \max }=u_{2 \max }=6$, and $k_{b}=0.2$.

The following are simulations for two kinds of stem valve opening degree conditions: within the limit and exceeding the limit.

(1) Mechanical power only appears $10 \%$ of the perturbation in a certain period of time (6s to $6.1 \mathrm{~s})$ and then returns to the known initial value, that is, $P+\Delta P(t)$, and the main valve control amplitude does not exceed the limit,

$$
\Delta P(t)= \begin{cases}0, & 0 \leq t<6 s \\ 0.1, & 6 \mathrm{~s} \leq t \leq 6.1 \mathrm{~s} \\ 0, & 6.1 \mathrm{~s}<t .\end{cases}
$$

Figures 3-6 show the state response curves of the system. The solid line denotes the response curve of the controller designed by Barrier Lyapunov, and the dotted line represents the response curve without considering state constraints. Figure 3 shows the condition of main valve control within the limit, in which the state response curve of $x_{1}$ is constrained. Visibly, the state is within the constraints. Moreover, the oscillation of the amplitude is also small for the other states. As shown in Figures 7, 9, and 10, which show the control input curve, the designed controller gain becomes larger with Barrier Lyapunov Function. The main valve control input does not exceed the limited amplitude, and the switching signal is keeping the value 1 , as shown in Figure 8. 


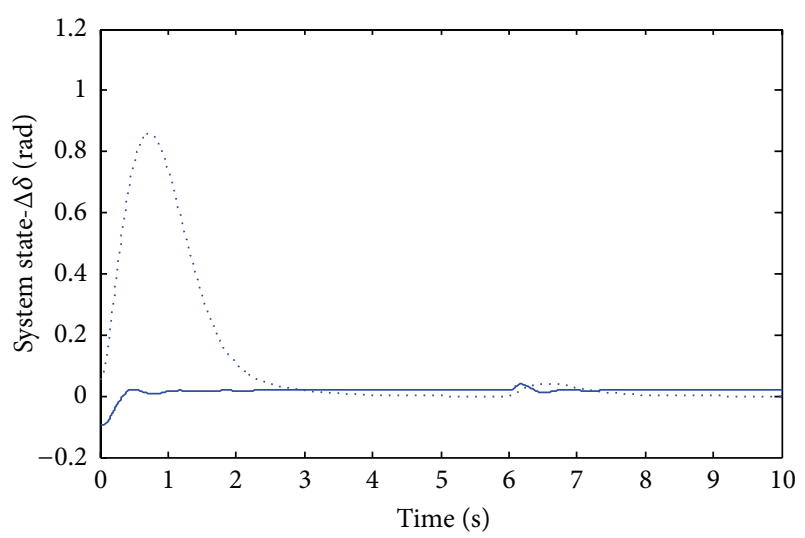

— Barrier Lyapunov Lyapunov

FIgURE 3: Transient response curves of the angle.

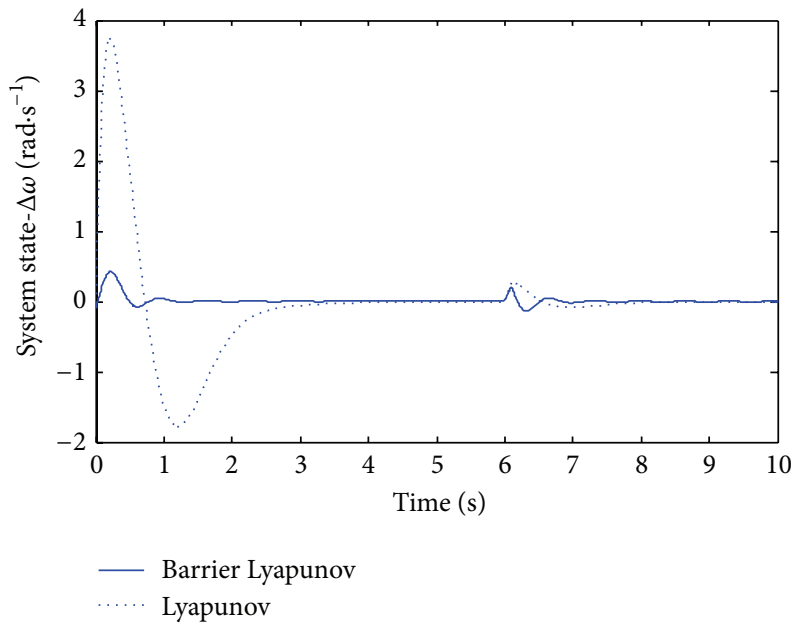

FIGURE 4: Transient response curves of the speed.

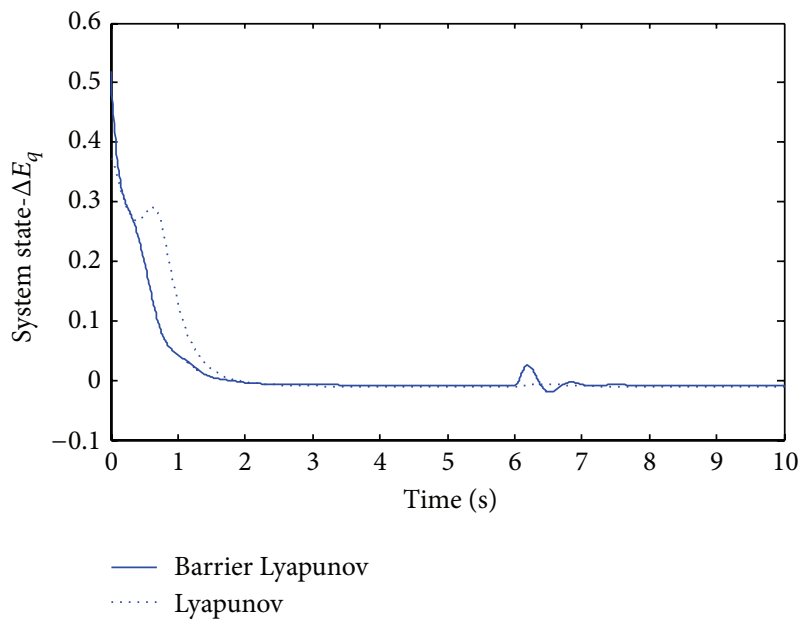

FIGURE 5: Transient response curve of the generator potential.

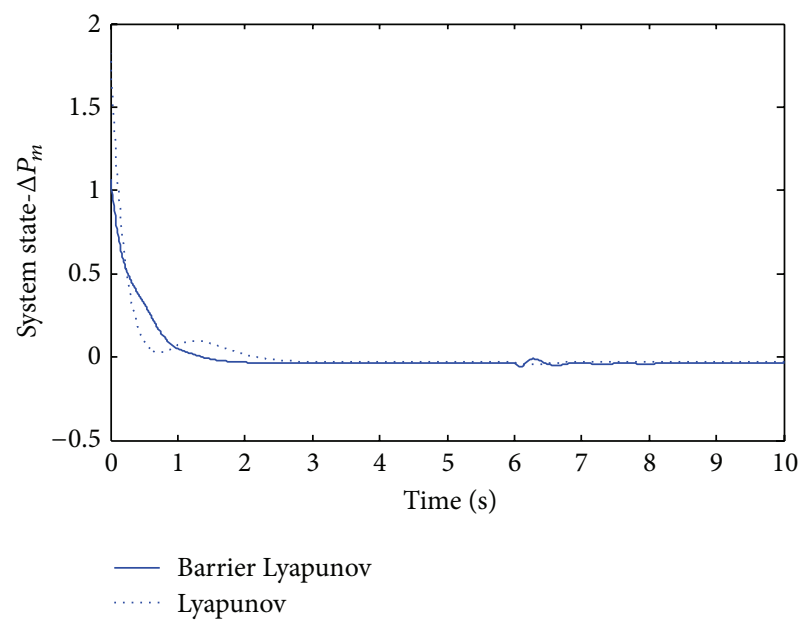

FIgURE 6: Transient response curve of the mechanical power.

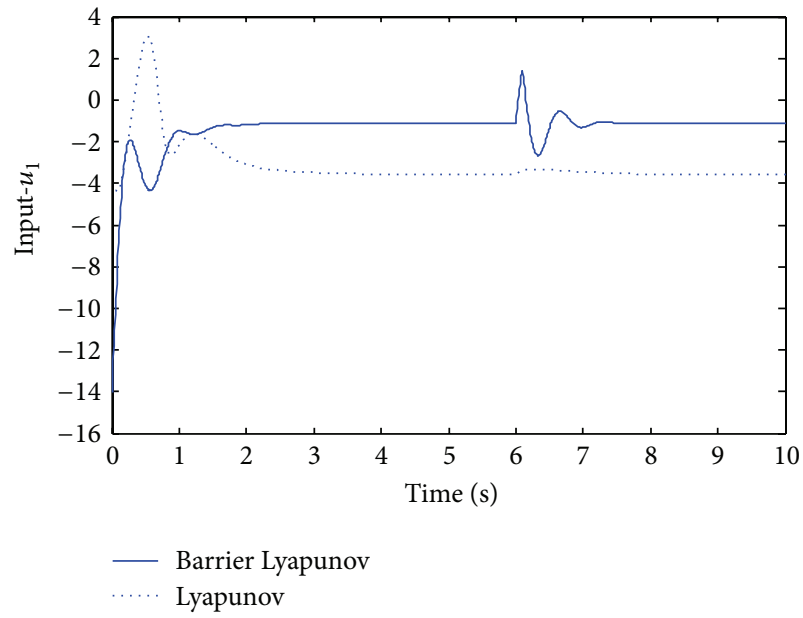

Figure 7: The excitation control input curves.

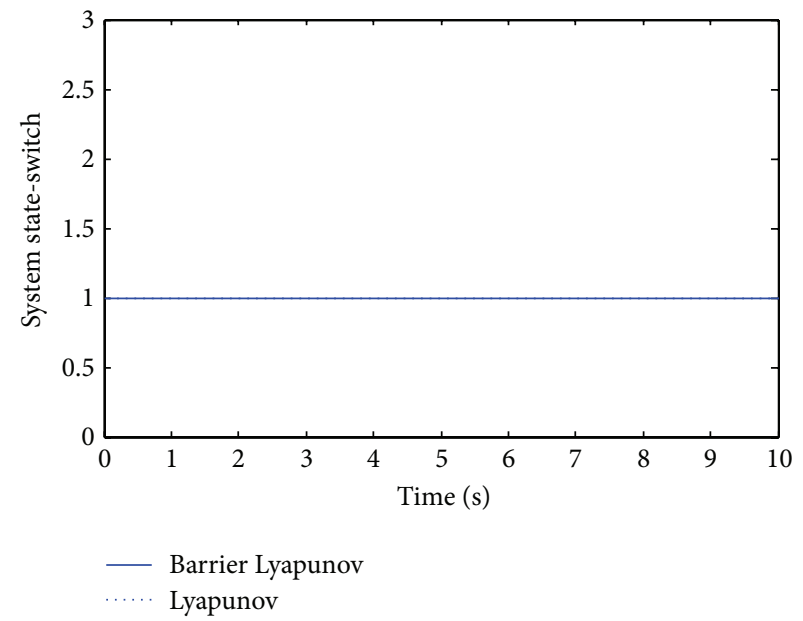

Figure 8: Switching signal. 


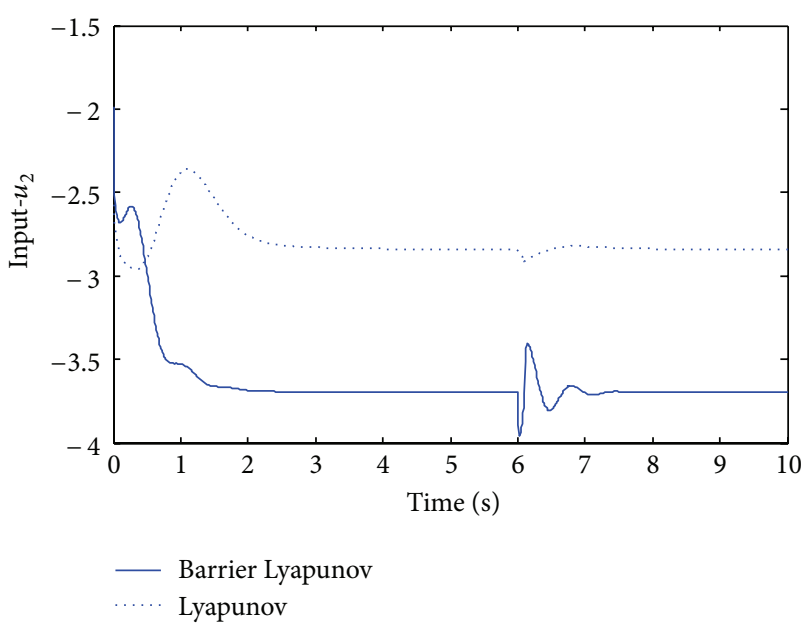

FIgURE 9: The main steam valve control input.

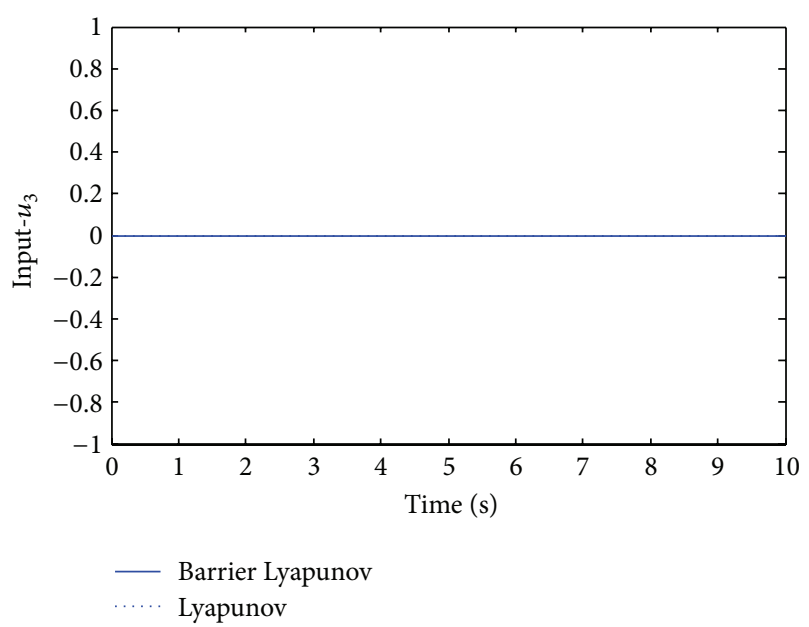

FIgURE 10: The fast steam valve control input.

(2) Mechanical power only appears $20 \%$ of the perturbation in a certain period of time (6s to $6.1 \mathrm{~s}$ ) and then returns to the known initial value, that is, $P+\Delta P(t)$, and the main valve control exceeds limited amplitude. Consider

$$
\Delta P(t)= \begin{cases}0, & 0 \leq t<6 \mathrm{~s} \\ 0.2, & 6 \mathrm{~s} \leq t \leq 6.1 \mathrm{~s} \\ 0, & 6.1 \mathrm{~s}<t\end{cases}
$$

Figures 11 to 14 show the state response curve of the system. The solid line denotes the response curve of the controller designed by Barrier Lyapunov, and the dotted line represents the response curve without considering state constraints. Figure 11 shows the condition of main valve control exceeding the limit, in which the state response curve of $x_{1}$ is constrained. Visibly, the state is within the constraints. Moreover, the oscillation of amplitude is also small for the other states. As shown in Figures 15, 17, and 18, which show the control input curve, the designed controller gain becomes larger with Barrier Lyapunov Function. When the main valve

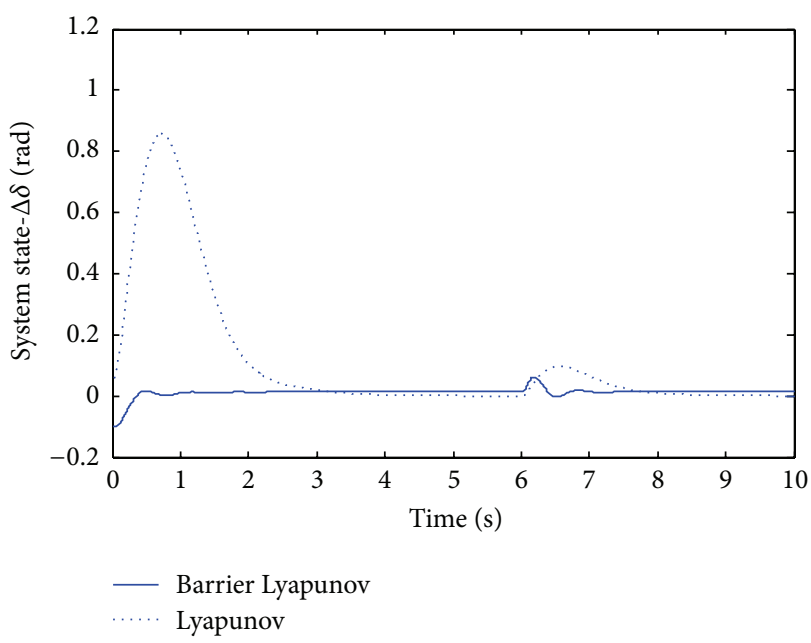

FIGURE 11: Transient response curves of the angle.

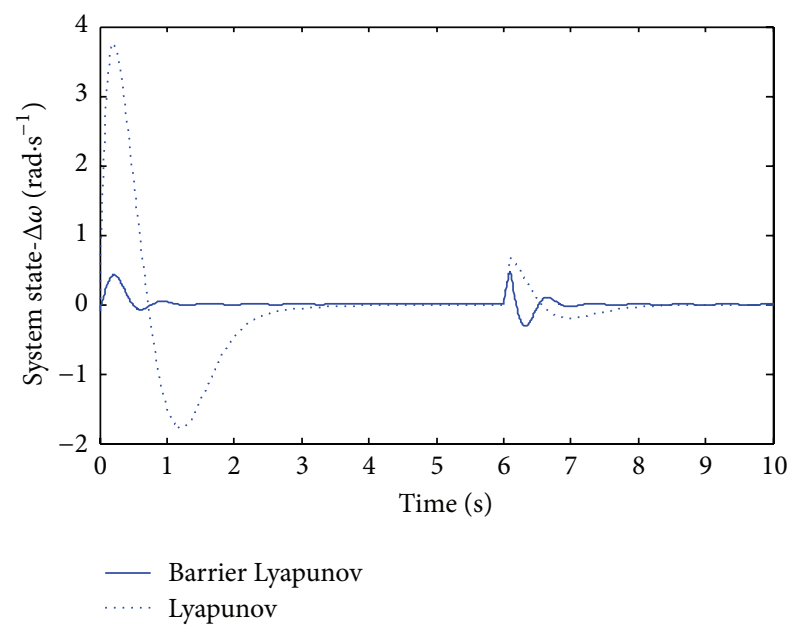

FIgURE 12: Transient response curves of the speed.

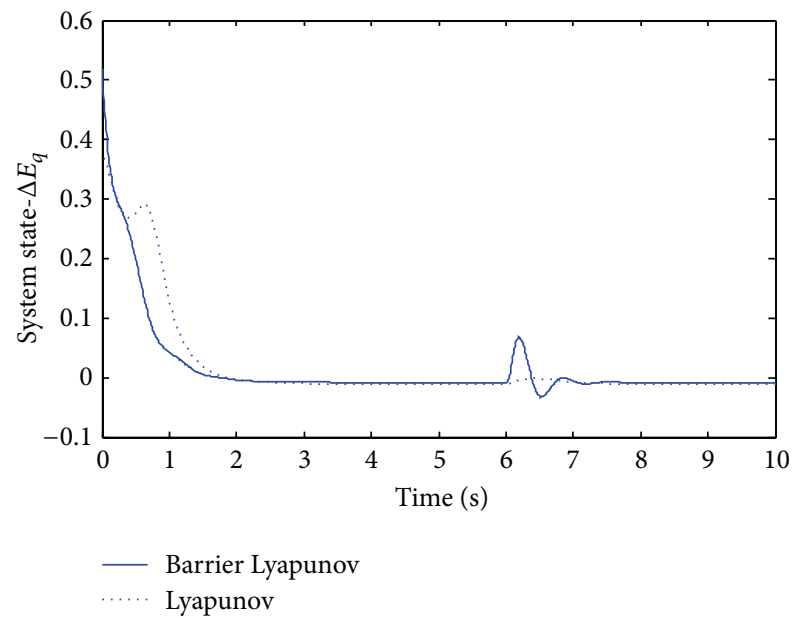

FIGURE 13: Transient response curve of the generator potential. 


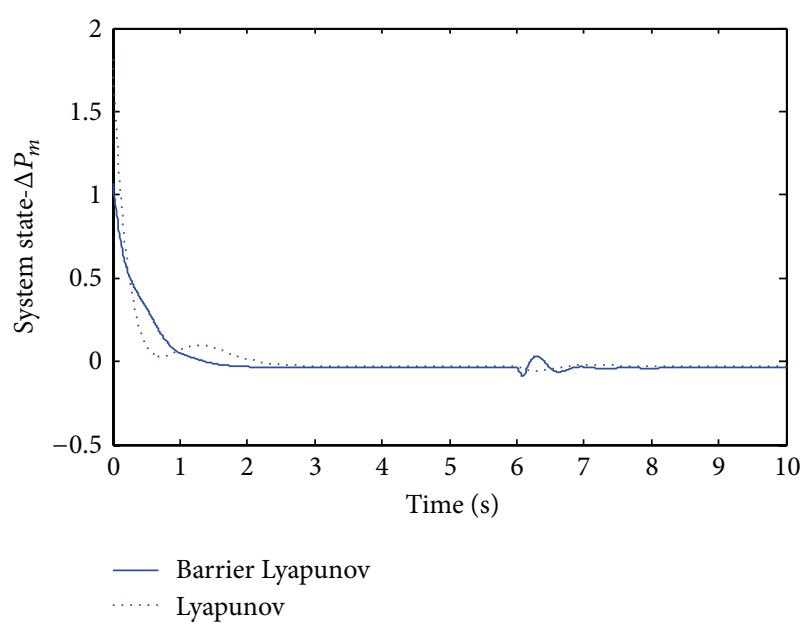

FIgURE 14: Transient response curve of the mechanical power.

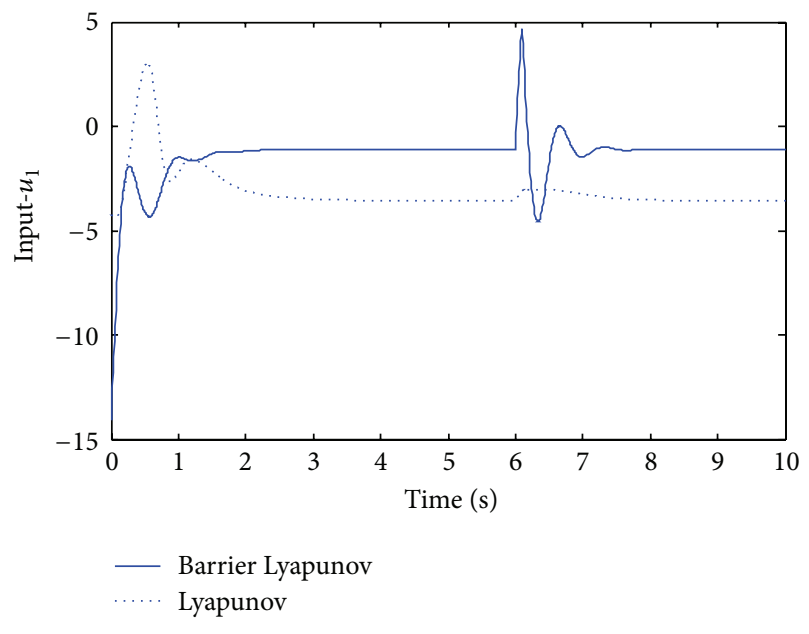

FIGURE 15: The excitation control input curves.

control input exceeds the limited amplitude, the switching signal changes from 1 to 2 , as shown in Figure 16. That is, when the main steam valve is overrun, its control input is 0 , and the fast valve starts to work. The valve control inputs are within the set range, without constraints destroyed.

\section{Conclusion}

In this paper, the coordinated excitation and valve controller of the steam turbine generator is designed by Barrier Lyapunov Function and backstepping method. Simulation results show that the generator power angle did not exceed the allowable value and the excitation and steam valve control gain is limited and within a reasonable range. With just requiring the initial power angle to satisfy certain conditions, we can conclude that the constrained state does not exceed the limit set by the constraint in the system. Thus, the design of the controller reduces the extent strictly required by the initial conditions. Furthermore, we can design a more effective controller and improve the efficiency of the power system.

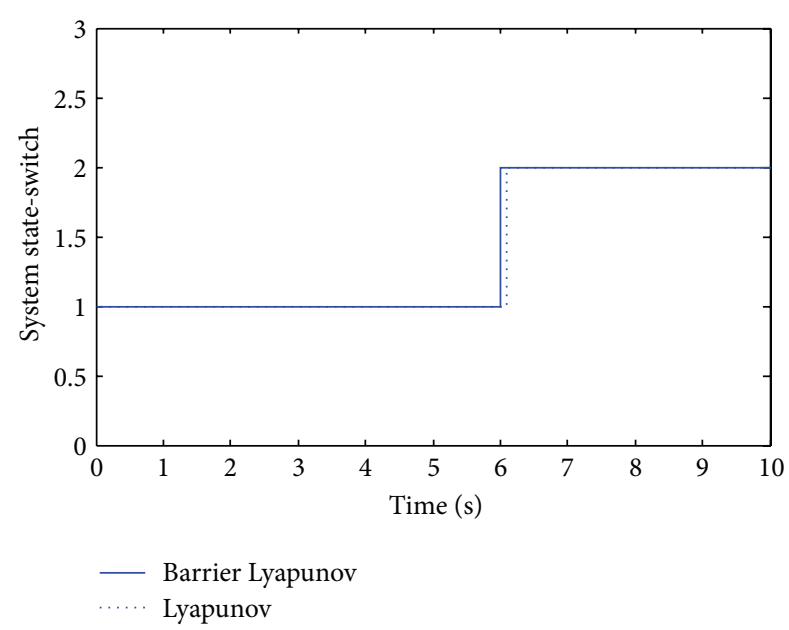

FIGURE 16: Switching signal.

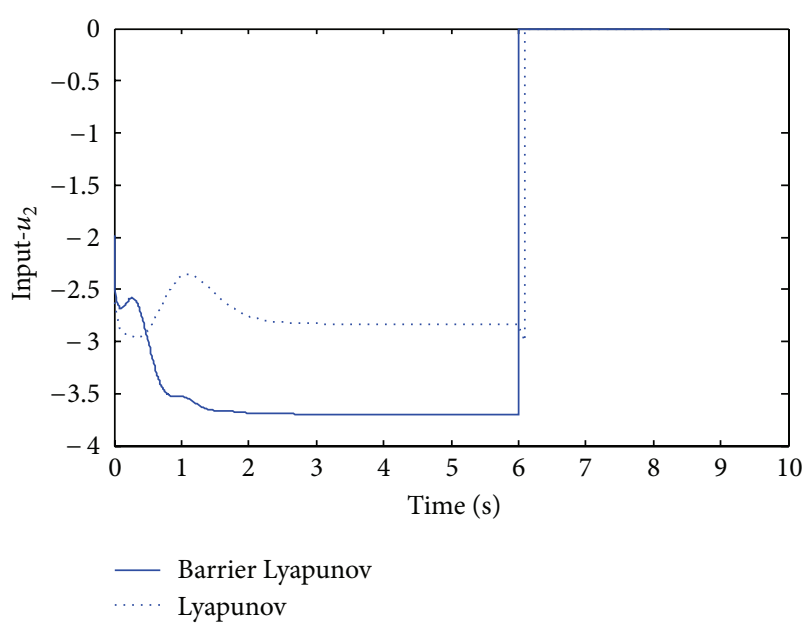

FIGURE 17: The main steam valve control input curves.

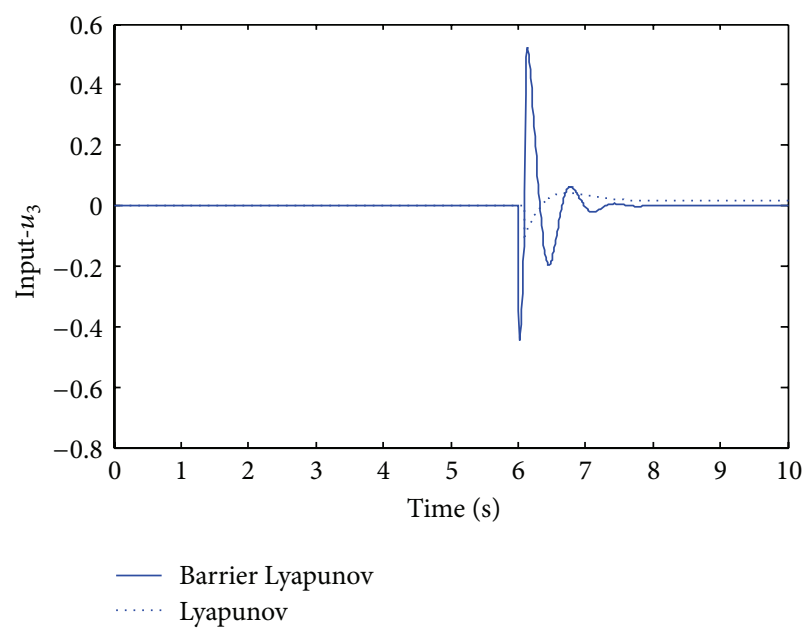

FIGURE 18: The fast steam valve control input curves. 


\section{Conflict of Interests}

The authors declare that there is no conflict of interests regarding the publication of this paper.

\section{Acknowledgments}

The authors would like to thank the anonymous reviewers for their helpful and insightful comments for further improving the quality of this work. This work is supported by the Fundamental Research Funds for the Central Universities, under Grant N130404022, and the National Natural Science Foundation of China, under Grants 61304021 and 61233002, and Specialized Research Fund for the Doctoral Program of Higher Education, under Grant 20020145007.

\section{References}

[1] W. Li, S. Liu, G. Jiang, and M. D. Geogi, "Adaptive robust backstepping design for turbine valve controller," in Proceedings of the 6th World Congress on Intelligent Control and Automation (WCICA '06), pp. 7439-7443, Dalian, China, June 2006.

[2] B. Ren, S. S. Ge, K. P. Tee, and T. H. Lee, "Adaptive neural control for output feedback nonlinear systems using a barrier Lyapunov function," IEEE Transactions on Neural Networks, vol. 21, no. 8, pp. 1339-1345, 2010.

[3] N. Jiang, X. Chen, T. Liu, B. Liu, and Y. Jing, "Nonlinear steam valve adaptive controller design for the power systems," Intelligent Control and Automation, vol. 2, no. 1, pp. 31-37, 2011.

[4] L.-Y. Sun and J. Zhao, "Nonlinear adaptive control for the turbine steam valve with input constraints," Control Theory \& Applications, vol. 26, no. 6, pp. 601-606, 2009.

[5] Y. Wan, Coordinated Control for Steam Turbine Generator Excitation and Steam Valve, Northeastern University, Shenyang, China, 2010.

[6] K. P. Tee and S. S. Ge, "Control of nonlinear systems with full state constraint using a barrier Lyapunov function," in Proceedings of the 48th IEEE Conference on Decision and Control Held Jointly with the 28th Chinese Control Conference (CDC/CCC '09), pp. 8618-8623, December 2009.

[7] Z. Li and L. Sun, "Nonlinear control for synchronous generator excitation system with output constraints," Journal of Liaoning Universtity of Technology, vol. 33, no. 3, pp. 141-144, 2013.

[8] N. Chang and Z. Guo, "Nonlinear decentralized robust valve controller constrained by control limits for turbo-generator," Relay, vol. 31, no. 12, pp. 1-4, 2003.

[9] M. Liu and L. Sun, "Nonlinear control for main steam valve of steam turbine generator unit with multiple state constraints," Journal of Liaoning Universtity of Technology, vol. 33, no. 6, pp. 351-355, 2013.

[10] K. D. Do, D. H. Nguyen, and T. B. Nguyen, "Nonlinear control of magnetic bearing," Journal of Measurement Science and Instrumentation, vol. 1, no. 1, pp. 10-16, 2010.

[11] K. B. Ngo, R. Mahony, and Z. Jiang, "Integrator Backstepping using barrier functions for systems with multiple state constraints," IEEE Conference on Decision and Control, vol. 8, no. 3, pp. 6-12, 2005.
[12] K. P. Tee, S. S. Ge, and E. H. Tay, "Barrier Lyapunov functions for the control of output-constrained nonlinear systems," Automatica, vol. 45, no. 4, pp. 918-927, 2009.

[13] G. X. Athanasius, H. R. Pota, P. B. Subramanyam, and V. Ugrinovskii, "Robust power system stabiliser design using minimax control approach: validation using real-time digital simulation," in Proceedings of the 46th IEEE Conference on Decision and Control, pp. 2427-2432, IEEE, New Orleans, La, USA, December 2007. 


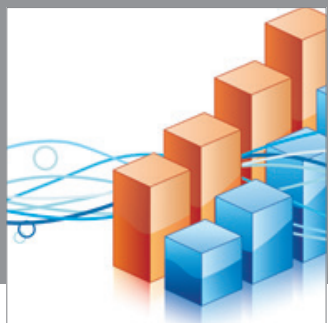

Advances in

Operations Research

mansans

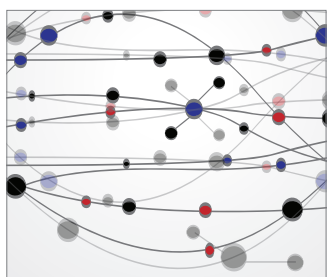

The Scientific World Journal
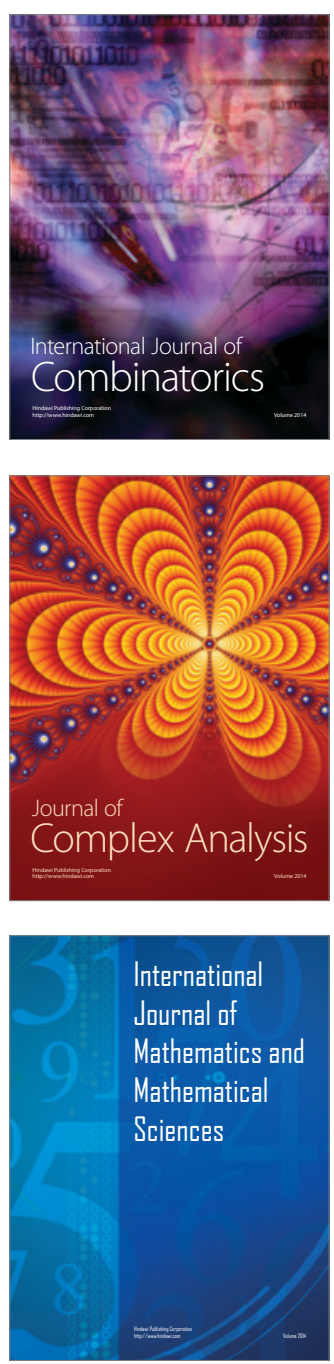
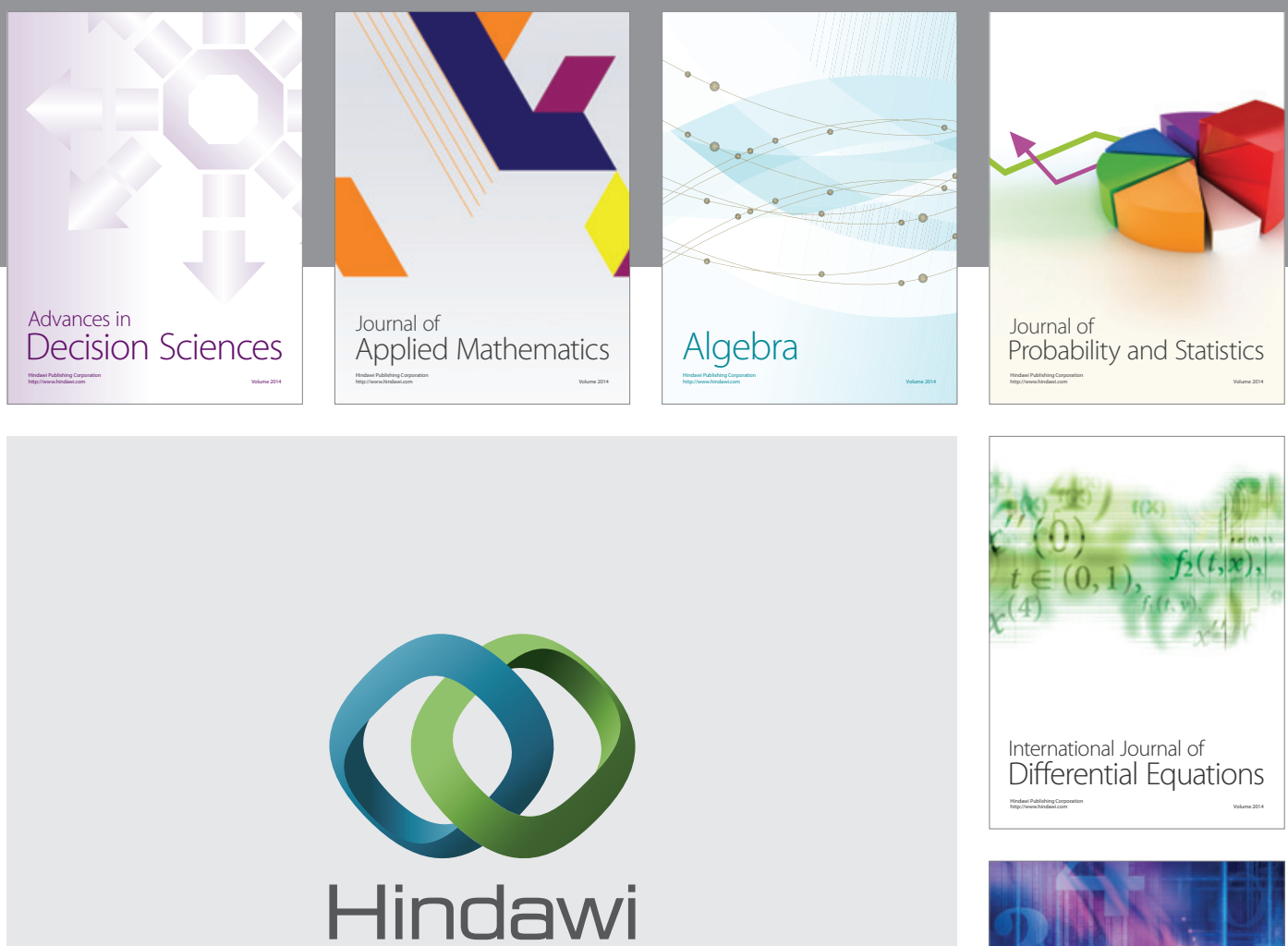

Submit your manuscripts at http://www.hindawi.com
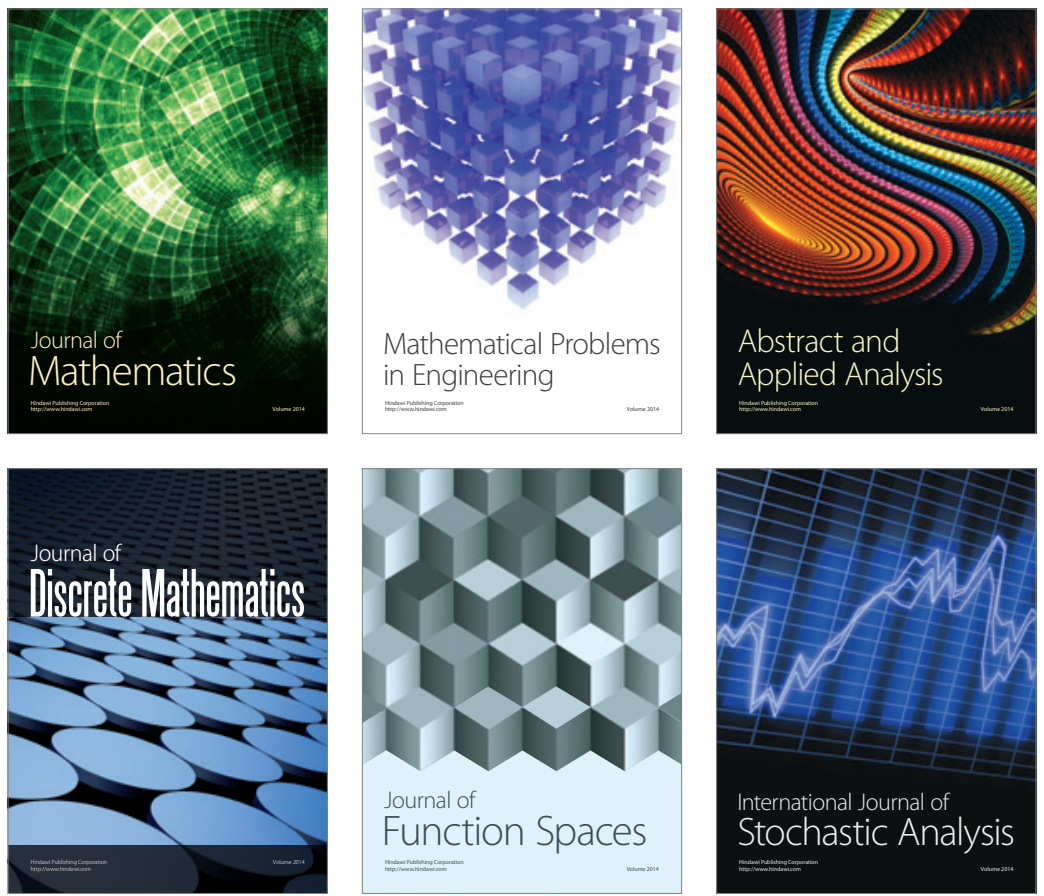

Journal of

Function Spaces

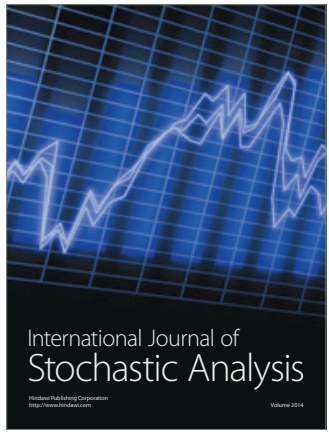

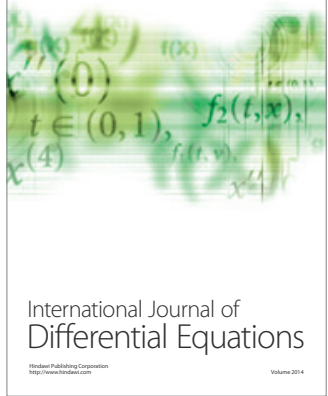
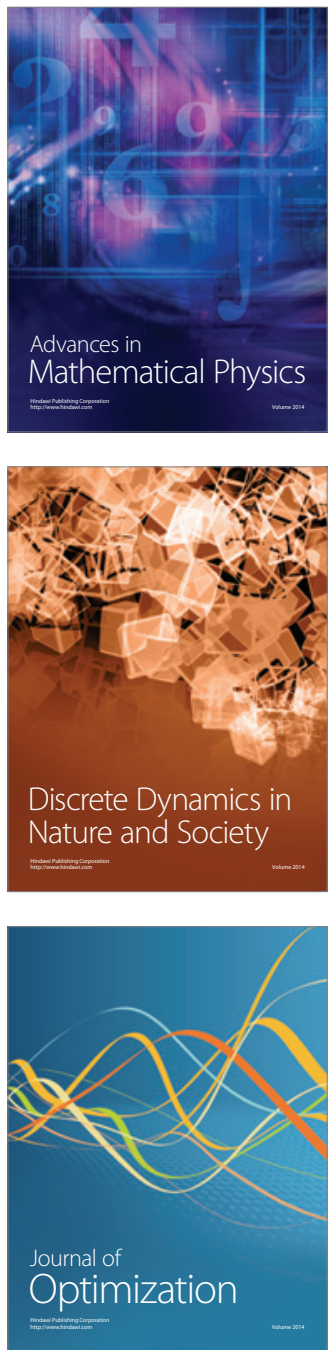